\title{
Mechanistic Models for hematological toxicities: small is beautiful
}

\author{
Laure Deyme $^{1}$, Sebastien Benzekry ${ }^{2}$, and Joseph Ciccolini ${ }^{3}$ \\ ${ }^{1}$ Aix-Marseille Universite \\ ${ }^{2}$ Institut National de Recherche en Informatique Appliquée \\ ${ }^{3}$ CRCM Inserm U1068 Aix Marseille Université
}

June 9,2020

In their interesting paper published in the British Journal of Clinical Pharmacology, Mackey et al. present an analysis aiming at characterizing resonance (i.e., neutrophils oscillations) in young patients treated by cyclic chemotherapy [1]. The authors used further Quantitative Systems Pharmacology (QSP) modeling applied to granulopoïesis to demonstrate that timing of chemotherapy could impact on the dynamics of neutrophils. Consequently, the authors suggest that model-informed scheduling could help limiting hematological toxicity, e.g. by delaying supportive G-CSF therapy. The issue of controlling drug-induced side effects, especially hematological toxicities with cytotoxics, is critical in many respects. Pancytopenia can be rapidly life-threatening, especially in frail patients. When they do not directly lead to toxic-death, such severe toxicities frequently oblige practitioners to postpone or discontinue chemotherapy or associated radiation therapy, thus affecting clinical outcome and survival eventually. In addition, severe neutropenia with sepsis require antibiotics which history of use is suspected now to compromise the efficacy of immune checkpoint inhibitors, probably because of disruption of gut microbiota [2]. In addition, it is now acknowledged that prolonged lymphopenia can have deleterious effect as well, especially in the era of immunotherapy. For instance, high neutrophils-tolymphocytes basal ratios have been repeatedly associated with poor response to immunotherapy because it could promote immune desert at the tumor level [3]. Altogether, developing strategies to control or reduce the risk of drug-induced hematological toxicities is therefore a major concern in clinical oncology, especially because as stated by the authors, cytotoxics are still today the backbone of most treatments of solid tumors and hematological malignancies. Developing in silico approaches as decision-making tools to optimize anticancer therapies has been a rising trend for decades now in clinical oncology [4]. Pharmacokinetically-guided regimen with Bayesian adaptive dosing procedures have been already proposed for several years to tailor the administration of a variety of cytotoxics and oral targeted therapies [5]. However, implementing adaptive dosing strategies in routine clinical setting remains challenging. Real-life precision medicine requires indeed mathematical models that are kept simple enough to allow proper identification of their parameters. This is a prerequisite for being easily applied prospectively in actual patients next, and not to be used solely as part of retrospective in silico modeling. This calls for using primarily top-down approaches, such as compartmental analysis prior to developing pharmacokinetics/pharmacodynamics (PK/PD) models likely to help oncologists to determine the optimal dosing and scheduling of a given drug to a given patient. More intricate modeling and QSP approaches are appealing strategies which are unfortunately impaired by their intrinsic complexity, which has made them unfit for routine use at bedside so far (Figure 1). Conversely, phenomenological modeling could in many respects look like an over-simplistic, suboptimal strategy, often mocked as being black-boxes simply linking an output to a given input. In turn, such models are more likely to be actually used in real-life setting, not despite the fact that they are black-boxes simply linking an output to a given input, but precisely because they are black-boxes simply linking an output to a given input. For instance, the Friberg model, as cited by the authors, is a simplified representation of hematopoiesis, using a semi-mechanistic, compartmental description of the proliferation and dynamics of the maturation of blood progenitors. Because of its simplicity, this top-down approach has allowed the Friberg model to 
be extensively used over the last 15 years, both by academics and pharmaceutical companies, to describe the myelosuppressive effects of a variety of cytotoxics [6]. Countless phenomenological models have been derived from the Friberg model ever since. For instance, the Meille model is based upon a similar simplified and semi-mechanistic representation of hematopoiesis and granulopoiesis, which encapsulates additionally a PK/PD model describing effects of supportive G-CSF administration on blood cells progenitors [7]. When further combined with another phenomenological model for antiproliferative efficacy, it was used next to build an original constraint-model determining the optimal dosing and scheduling of densified chemotherapy combo plus G-CSF support. Once calibrated with pre-defined acceptable levels of hematological toxicity and desired level of tumor shrinkage, this mathematical-driven regimen was finally tested prospectively in metastatic breast cancer patients and showed excellent performances such as prolonged overall survival in heavily pretreated patients with fully controlled hematological toxicities [8]. Importantly, the prospective use of such a mathematical model was made possible thanks to a first identification step with few blood samples taken from patients when treatment starts, providing individual data on drugs PK profile and blood counts . This critical step allowed fine tuning of individual PK/PD model parameters in real-time, thus ensuring optimal, personalized dosing next. Transposing such model-driven regimen at bedside seems to be only achievable when the whole modeling strategy is primarily built upon the parsimony principle, so as to be able to identify next individual parameters from sparse, routine data collected from patients in a reallife setting. Of note, no in-depth understanding of biological mechanisms can be provided by such models - dosing and scheduling of anticancer agents and G-CSF are connected to efficacy and toxicity endpoints through phenomenological black-boxes, not multi-scales models providing biological explanations of the phenomenon. In contrast, QSP models, such as the ones developed by the Craig group in this BJCP issue, offer appealing mechanistic features, thus allowing a better understanding of all the underlying mechanisms at play to explain pharmacodynamic endpoints. The downside is that the Mackey model is complex. It is based indeed upon more than 30 parameters, a large number of them having been fixed from the literature and thus are dependent on the variability and possible biases of the experiments used for their identification [9]. Furthermore, in contrast to standard approaches in pharmacometrics using nonlinear mixed-effects (NLME) modeling, inter-individual variability of the parameters is not quantified. The issue with such a large number of parameters is that the practical identifiability from sparse individual data collected at bedside is expected to be poor, resulting in a large uncertainty for quantitative model predictions in real-life practice.

Nevertheless, the qualitative observation of the resonance effect in neutrophils time dynamics induced by the administration of periodic chemotherapies, highlighted by Mackey et al., should prompt modelers to include such pivotal phenomenon, including in their phenomenological representations of hematological toxicity. This new concept could be easily integrated and quantitatively tested in NLME models by means of inter-occasion variability (IOV) between administrations. Such IOV could allow to describe the resonance effect and improve model predictions. A covariate analysis could be performed to identify potential factors explaining the origin of this observation. Furthermore, as stated by the authors, to avoid this deleterious phenomenon and to reduce cytotoxic-induced hematological toxicities with subsequent negative impact on tumor immunity, it is an absolute prerequisite to optimize empirical chemotherapy regimens and G-CSF support administrations, based on robust PK/PD compartmental modeling.

Altogether, the Mackay study highlights how the very way we use anticancer agents does matter, and how a same drug can have diametrically different pharmacodynamics effects, depending on its scheduling. In this respect, this work is an important contribution to the field of Precision Medicine in Oncology, by suggesting that there is much room left to improve standard use of canonical cytotoxics and thatin silico strategies could help to achieve a better way to administrate anticancer drugs. To transform this theoretical concept into a practical decision-making tool for oncologists, mathematicians and modelers must compose with the issue of parameters identifiability, and what kind of accessible individual data are actually made available in routine patients. As long as these issues are nor fixed, modeling will remain an elegant but theoretical field disconnected from bedside practice. To the ever-rising complexity of cancer biology and the amazing amount of knowledge regarding in-depth mechanisms of action of drugs (such as molecular signaling pathways, genetic and epigenetic regulations affecting targets or key-proteins involved in pharmacodynamics responses), the 
temptation to implement all this knowledge into super-models should be resisted. Indeed for a proper and rapid in silico -to-bedside transposition, we believe that in the era of Precision Medicine, the more complex is a phenomenon, the simpler should be the mathematical model describing it.

Competing Interest: the authors declare none

Funding: none

\section{References}

[1] Mackey M, Glisovic S, Pastore Y, Krajinovic M, Craig M. The timing of cyclic cytotoxic chemotherapy can worsen neutropenia and neutrophilia. Brit J Clin Pharmacol 2020; EPub Ahead of Print.

[2] Derosa L, Hellmann MD, Spaziano M, Halpenny D, Fidelle M, Rizvi H, Long N, Plodkowski AJ, Arbour KC, Chaft JE, Rouche JA, Zitvogel L, Zalcman G, Albiges L, Escudier B, Routy B. Negative association of antibiotics on clinical activity of immune checkpoint inhibitors in patients with advanced renal cell and non-small-cell lung cancer. Ann Oncol 2018; 29: 1437-1444.

[3] Sacdalan DB, Lucero JA, Sacdalan DL. Prognostic utility of baseline neutrophil-to-lymphocyte ratio in patients receiving immune checkpoint inhibitors: a review and meta-analysis. Onco Targets Ther 2018 23; 11:955-965

[4] Barbolosi D, Ciccolini J, Lacarelle B, Barlési F, André N. Computational oncology-mathematical modelling of drug regimens for precision medicine. Nat Rev Clin Oncol 2016; 13:242-54.

[5] Ferrer F, Fanciullino R, Milano G, Ciccolini J. Towards Rational Cancer Therapeutics: Optimizing Dosing, Delivery, Scheduling, and Combinations Clin Pharm Ther 2020; EPub Ahead of Print.

[6] Friberg LE, Karlsson MO. Mechanistic Models for Myelosuppression. Investigational New Drugs 2003; 21: 183-194.

[7] Meille C, Barbolosi D, Ciccolini J, Freyer G, Iliadis A. Revisiting Dosing Regimen Using Pharmacokinetic/Pharmacodynamic Mathematical Modeling: Densification and Intensification of Combination Cancer Therapy. Clin Pharmacokinet 2016; 55:1015-25.

[8] Hénin E, Meille C, Barbolosi D, You B, Guitton J, Iliadis A, Freyer G. Revisiting dosing regimen using PK/PD modeling: the MODEL1 phase I/II trial of docetaxel plus epirubicin in metastatic breast cancer patients. Breast Cancer Res Treat 2016; 156: 331-41.

[9] Craig M, Humphries AR, Mackey M. mathematical model of granulopoiesis incorporating the negative feedback dynamics and kinetics of G-CSF/neutrophil binding and internalisation. Bull Math Biol 2016; 78: 2304-2357.

\section{Figure 1.}

Pros and Cons of Top-down VS. Bottom-up strategies in clinical oncology. 


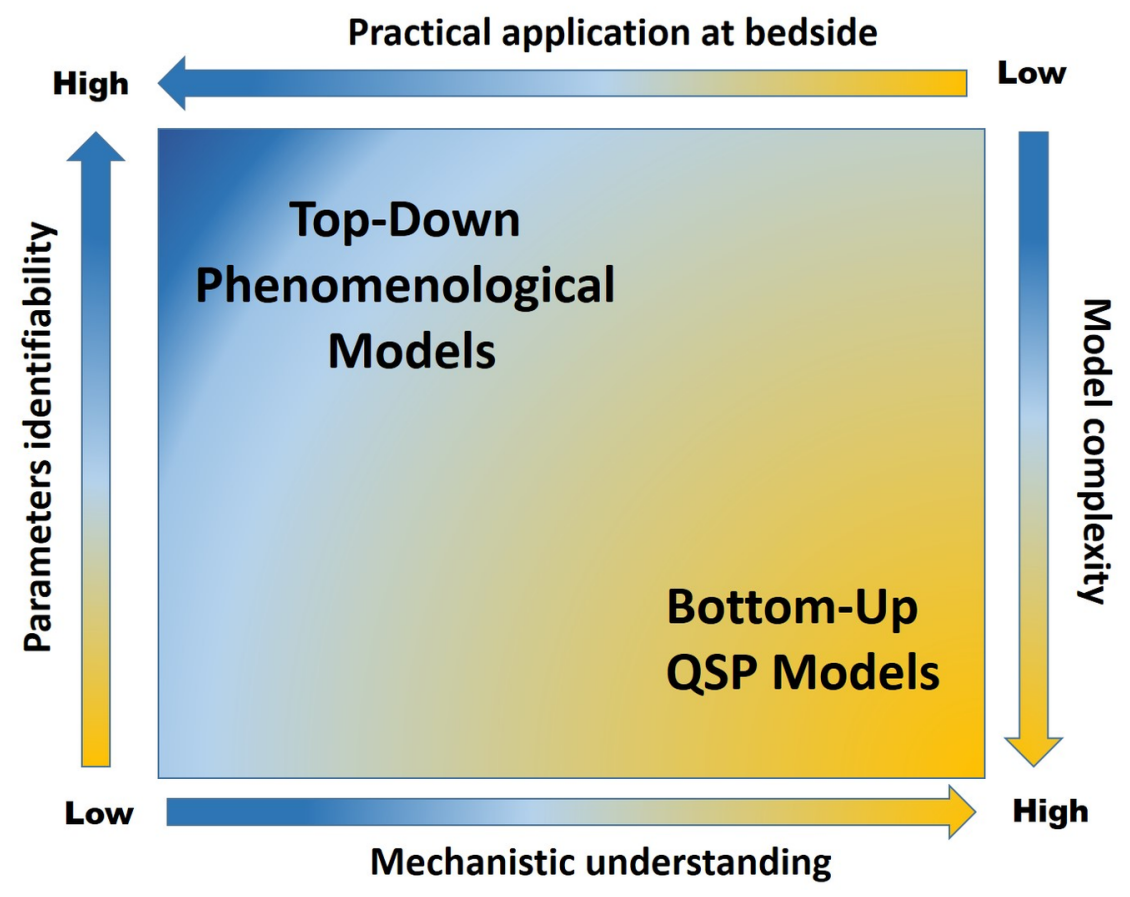

